\title{
A new mutation in the AFP gene responsible for a total absence of alpha feto-protein on second trimester maternal serum screening for Down syndrome
}

\author{
François M Petit ${ }^{1}$, Marylise Hébert ${ }^{1}$, Olivier Picone ${ }^{2}$, Sophie Brisset ${ }^{3}$, Marie-Laure Maurin ${ }^{3}$, \\ Frédéric Parisot ${ }^{1}$, Liliane Capel ${ }^{1}$, Clarisse Benattar ${ }^{1}$, Marie-Victoire Sénat ${ }^{2}$, \\ Gérard Tachdjian ${ }^{3}$ and Philippe Labrune ${ }^{4}$
}

${ }^{1}$ Department of Biochemistry, Hormonology and Genetics, Antoine Béclère Hospital AP-HP, Université Paris Sud, Clamart,
France; ${ }^{2}$ Department of Gynaecology and Obstetrics, Antoine Béclère Hospital AP-HP, Université Paris Sud, Clamart,
France; ${ }^{3}$ Department of Cytogenetics, Antoine Béclère Hospital AP-HP, Université Paris Sud, Clamart, France;
${ }^{4}$ Department of Paediatrics and Medical Genetics, Antoine Béclère Hospital AP-HP, Université Paris Sud, Clamart, France

Alpha feto-protein (AFP) is a major plasma protein produced by the yolk sac and the liver during the fetal period. During the second trimester of pregnancy, APF and $\beta \mathrm{hCG}$ serum concentrations are commonly used for screening Down syndrome. AFP deficiency is rare (estimated to be 1/105000 newborns) and only one sequence alteration has previously been reported in the AFP gene. We report a new mutation in exon 5 of the AFP gene, leading to a total absence of AFP on 2nd-trimester maternal serum screening for Down syndrome, confirmed on the amniotic fluid. Despite this, fetal development and birth were normal. After PCR-amplification, the whole AFP gene was sequenced. The new mutation was a guanine to adenine transition in position 543 creating a premature stop codon in position 181. In order to search for eventual modifications of the amniotic fluid profile, proteins were separated by electrophoresis and compared with 10 normal amniotic fluids sampled at the same developmental age (18 weeks). In the amniotic fluid of our patient albumin rate was reduced whereas alpha1 and beta protein fractions were increased, suggesting that AFP deficiency may modify the distribution of protein fractions. This observation emphasizes the complex molecular mechanisms of compensation of serum protein deficiency. Studies on other families with AFP deficiency are necessary to confirm this observation.

European Journal of Human Genetics (2009) 17, 387-390; doi:10.1038/ejhg.2008.186; published online 15 October 2008

Keywords: congenital AFP deficiency; Down syndrome screening; protein regulation

\section{Introduction}

Alpha feto-protein (AFP) is a major plasma protein produced by the yolk sac and the liver during the fetal

Correspondence: Dr FM Petit, Department of Biochemistry, Hormonology and Genetics, Antoine Béclère Hospital, 157 rue de la Porte de Trivaux, 92141 Clamart Cedex, France.

Tel: + 331453743 09; Fax: + 331453747 45;

E-mail: francois.petit@abc.aphp.fr

Received 14 May 2008; revised 31 July 2008; accepted 11 September 2008; published online 15 October 2008 period. This fetal protein diffuses in maternal circulation through the placenta and can be detected in the maternal plasma. During the second trimester of pregnancy, serum APF and hCG are commonly used for evaluating the risk of Down syndrome. ${ }^{1}$ Low and high levels of AFP are indicative of Down syndrome and spina bifida, respectively. After birth, the AFP gene expression dramatically decreases to generally undetectable levels. Molecular mechanisms of protein transition between AFP and albumin during the fetal period are currently not completely understood. 
AFP gene is located on chromosome 4 and is a member of a multigenic family encoding several plasma proteins, such as albumin or vitamin D-binding protein. To date, rare cases of AFP deficiency during fetal life have been reported: 15 in France, ${ }^{2} 4$ in Israel, ${ }^{3-5} 2$ in the United States. ${ }^{6}$ Congenital deficiency was genetically confirmed in only one case. ${ }^{5}$ The authors identified the deletion c.882_883delCT at the homozygote state in two unrelated families.

Here, we report the case of a family originating from Algeria, in whom a new nucleic substitution in the exon 5 of the AFP gene was identified resulting in AFP deficiency.

\section{Patients and methods}

The mother was 27 years old and was nulligest. Her first pregnancy was obtained by intrauterine insemination, owing to asthenozoospermia. At 12 weeks, fetal ultrasound examination showed a single embryo measuring $60 \mathrm{~mm}$ (crown-to-rump length) and $1 \mathrm{~mm}$ for nuchal translucency. At 14 weeks and 1 day, second trimester maternal serum screening for Down syndrome was performed measuring AFP and hCG. hCG rate was $237641 \mathrm{IU} / 1$ (4.74 MoM) and AFP was undetectable $(<0.06 \mathrm{MoM})$, resulting in a very high risk of Down syndrome $(>1 / 10)$. At 15 weeks, fetal ultrasound examination was normal, but at 18 weeks, nasal bones were not seen. Amniocentesis was performed for both chromosomal analysis and biochemical assays. Cytogenetic investigations showed a normal male karyotype 46,XY, without mosaicism. In amniotic fluid, AFP was also undetectable. Fetal ultrasound examinations were performed at weeks 22 and 32 and proved normal. Birth occurred at 37 weeks after rupture of the amniochorionic membrane. The newborn weighted $3050 \mathrm{~g}$ and measured $51 \mathrm{~cm}$. He was transferred in the neonatology unit for mild heart rhythm abnormalities. At 4 days, both mother and neonate were discharged. Development and growth were normal.

Alpha feto-protein (measured pure in duplicate) and hCG (diluted at 1/100) level determinations were both performed by immunochemistry (AdviaCentaur immunoassay system, Bayer Healthcare). Briefly, AFP or hCG were picked by monoclonal antibodies bound to paramagnetic particles and revealed with polyclonal antibodies linked to acridinium ester. Thresholds of detection for the two markers were $1.3 \mathrm{ng} / \mathrm{ml}(1.08 \mathrm{IU} / \mathrm{ml})$ and $2 \mathrm{IU} / \mathrm{l}$, respectively. Risk of Down syndrome was calculated using T21 Bayer Diagnostics software (Bayer Healthcare).

Fetal DNA was extracted from cultured amniocytes using QIAamp DNA mini kit (QiaGen, Courtabœuf, France). Primers were designed for amplificating the 14 coding exons of the $A F P$ gene (including the intron-exon junctions), using Primer3 (Primer3 website: http://frodo.wi.mit. edu/cgi-bin/primer3/primer3_www.cgi). Both strands of each exon was sequenced on an ABI Prism 3130 sequencer (AppliedBiosystems, Courtaboeuf, France) and compared with the AFP gene reference (NC_000004.10) sequence using SeqScape 2.0 software (Applied Biosystems).

Total proteins and albumin rates were determined on amniotic fluid using photometry method (pyrogallol red-molybdate method, BioMérieux, Lyon, France) and nephelometry (Dade Behring, Marburg, Germany), respectively. After concentration by Minicon B15 (Millipore, Carrigtwohill, Ireland), amniotic proteins were separated by electrophoresis onto agarose gel (Sebia, Evry, France) and protein fractions were determined with Hydrasis 2 detection system (Sebia). Mean (m) and standard deviation $(\sigma)$ were calculated from a panel of 10 normal amniotic fluids sampled at 18 weeks (amniocentesis for age; no chromosomal abnormality detected).

\section{Results}

The sequence of the 14 coding exons of the AFP gene and their flanking regions was compared with the reference sequence. A homozygote nonsense mutation c.543G $>$ A in exon 5 was identified, resulting in the changing of the tryptophan in position 181 to a stop codon at the protein level (p.Trp181X) (Figure 1).

Total amniotic proteins rate, albumin rate and amniotic protein fraction repartition are presented in Table 1. Compared with the 10 controls, albumin fraction was underrepresented $(<\mathrm{m}-2 \sigma)$, whereas alpha1 and beta protein fractions were overrepresented $(>\mathrm{m}+2 \sigma)$. The other protein fractions, total proteins and albumin were not different from controls.

\section{Discussion}

We report a homozygote guanine-to-adenine substitution at nucleotide 543 in exon 5 of the $A F P$ gene (c.543G >A) in an Algerian family, whereas serum and amniotic AFP were

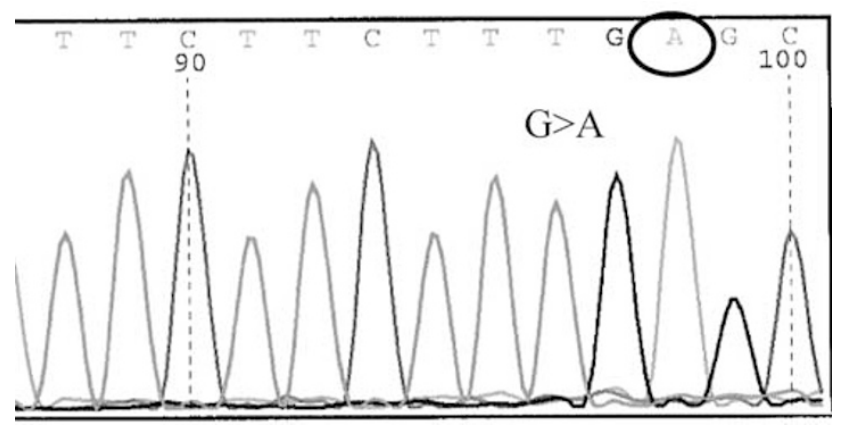

Figure 1 Sequence of exon 5 in our patient showing the homozygote mutation c.543G $>$ A creating a premature opal stop codon in position 181 (TGA). 
Table 1 Amniotic fluid protein profiles for this case and the 10 controls

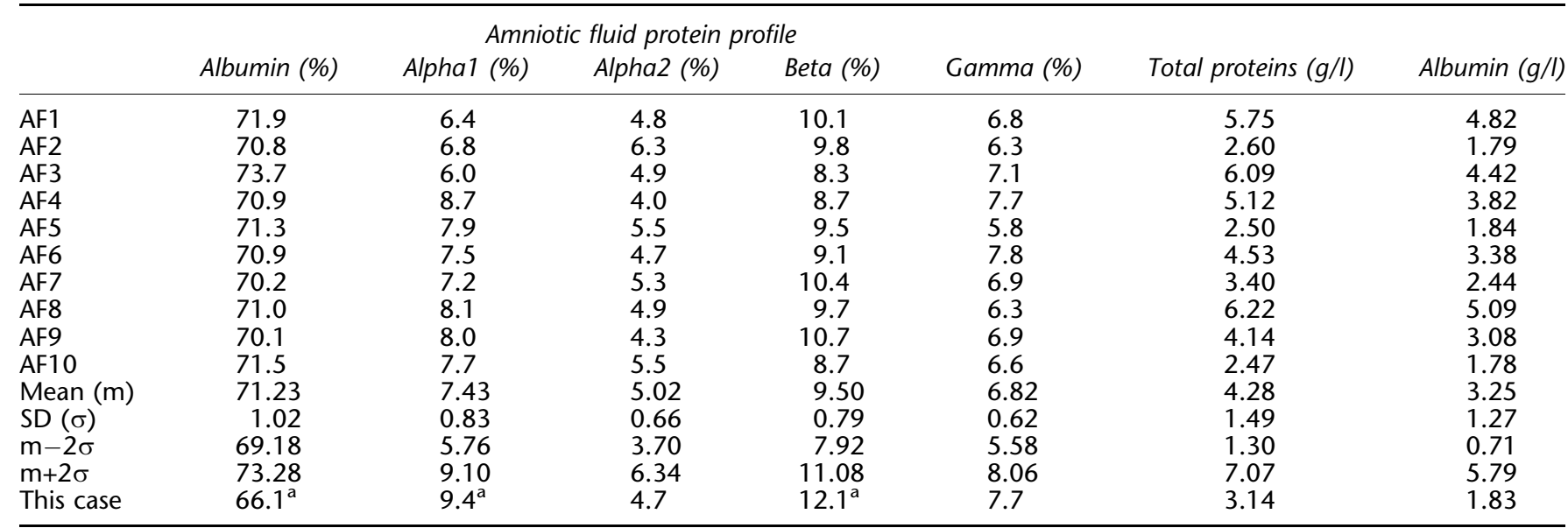

Mean $(\mathrm{m})$ and SD (standard deviation, $\sigma$ ) are calculated from the values measured in the 10 controls (AF1-AF10).

andicate values out of the range $m+/-2 \sigma$.

undetectable during the second trimester maternal serum screening for Down syndrome. This nonsense mutation was most likely to be responsible for a reduction of AFP mRNA stability, thus resulting in a premature RNA degradation, but this hypothesis could not be verified. The implication of this mutation in the phenotype of null production of AFP was not determined by in vitro expression studies, but convincing arguments support this relation. First, no AFP was detected in both maternal serum and amniotic fluid using immunological detection methods even though high AFP levels are usually observed. Secondly, the c.543G $>$ A mutation was the only nonsynonymous sequence abnormality observed in the 14 sequenced exons of the AFP gene. Unfortunately, the presence of the mutation at the heterozygote state in the parents could not be confirmed (blood sample refused).

Low or undetectable level of AFP during gestation is a rare condition. Its frequency was estimated to be $1 /$ $105000 .^{2}$ In such situations, the difficulty is to distinguish real low AFP values in patients at high risk of maternal or fetal abnormalities ${ }^{7}$ from congenital absence of AFP. $^{3}$ Indeed, molecular defect in the AFP gene expression could conceal 1 of the 18 clinical manifestations observed in case of low levels of serum AFP. ${ }^{7}$ In our case, these clinical manifestations have been excluded thanks to cytogenetic and biochemical investigations and to ultrasound examinations.

Congenital AFP deficiency is always considered as a benign trait, but this specific point may need to be modulated. Indeed, congenital AFP deficiency is generally diagnosed on second trimester maternal serum screening for Down syndrome, in other words, lately in the fetal development. To illustrate this interrogation, analbuminemia is a very rare metabolic abnormality considered as remarkably benign in infancy, but it could be responsible for severe disorders during intrauterine development (intrauterine growth retardation and intrauterine death). ${ }^{8}$ In this way and knowing its potential role in fetal immune function and in maintenance of osmotic pressure, AFP deficiency should be responsible for precocious fetal loss in some cases in which the diagnosis is not established. Molecular analyses of the AFP gene in precocious pregnancy loss are necessary to elucidate this specific point.

The mechanisms of the switch between AFP and albumin at the end of the embryonic period are not completely elucidated. In mouse, AFP and albumin mRNAs accumulate in parallel during the prenatal period, not only in hepatic cells but also in other tissues, suggesting a systematic concomitant expression of both genes. ${ }^{9}$ According to this hypothesis, albumin can replace AFP since the first developmental stages. Albumin rate and total protein fraction repartition differences between our case and controls suggest the implication of other proteins than albumin in the compensating mechanisms of AFP deficiency. However, this observation has to be interpreted with precaution because we studied only one case and total proteins and albumin measured in the control amniotic fluids were different from one another, probably due to variation in renal functions. Several studies on AFP deficiency are necessary for a better understanding of the mechanisms of compensation of the absence of AFP.

Although AFP deficiency is considered as benign, molecular analysis in case of total absence of AFP on second trimester maternal serum screening for Down syndrome should be systematic to inform the patient and his family for an accurate interpretation of AFP monitoring should hepatocellular carcinoma or testis tumors occur. 


\section{References}

1 Heyl PS, Miller W, Canick JA: Maternal serum screening for aneuploid pregnancy by alpha-fetoprotein, hCG, and unconjugated estriol. Obstet Gynecol 1990; 76: 1025-1031.

2 Muller F, Dreux S, Sault C et al: Very low alpha-fetoprotein in Down syndrome maternal serum screening. Prenat Diagn 2003; 23: 584-587.

3 Sher C, Shohat M: Congenital deficiency of AFP and Down syndrome screening. Prenat Diagn 1997; 17: 884-885.

4 Sharony R, Amiel A, Bouaron N, Kidron D, Itzhaky D, Fejgin M: Congenital deficiency of alpha-fetoprotein and associated chromosomal abnormality in the placenta. Am J Med Genet A 2003; 121: 113-117.
5 Sharony R, Zadik I, Parvari R: Congenital deficiency of alpha fetoprotein. Eur J Hum Genet 2004; 12: 871-874.

6 Greenberg F, Faucett A, Rose E et al: Congenital deficiency of alpha-fetoprotein. Am J Obstet Gynecol 1992; 167: 509-511.

7 Mizejewski GJ: Levels of alpha-fetoprotein during pregnancy and early infancy in normal and disease states. Obstet Gynecol Surv 2003; 58: 804-826.

8 Koot BG, Houwen R, Pot DJ, Nauta J: Congenital analbuminaemia: biochemical and clinical implications. A case report and literature review. Eur I Pediatr 2004; 163: 664-670.

9 Tilghman SM, Belayew A: Transcriptional control of the murine albumin/alpha-fetoprotein locus during development. Proc Natl Acad Sci USA 1982; 79: 5254-5257. 